\title{
Robust Streaming Erasure Codes using MDS Constituent Codes
}

\author{
Ahmed Badr, Ashish Khisti \\ School of Electrical and Computer Engineering \\ University of Toronto \\ Toronto, ON, M5S 3G4, Canada \\ \{abadr, akhisti\}@comm.utoronto.ca
}

\author{
Wai-Tian Tan, John Apostolopoulos \\ Mobile and Immersive Experience Lab \\ Hewlett Packard Laboratories \\ Palo Alto, California, 94304 \\ \{wai-tian.tan, japos\}@hp.com
}

\begin{abstract}
We revisit two classes of previously proposed robust streaming-erasure codes - MIDAS codes and PRC codes which guarantee streaming recovery over a class of packet erasure channels. We propose a modified construction for each class using diagonally-interleaved MDS codes. Our proposed codes have near identical performance over deterministic erasure channels, but only require a field size that grows polynomially in the delay. In contrast the previous constructions require that the field size increase exponentially with the delay. We also evaluate the performance of the proposed codes over GilbertElliott channels and show that some penalty does result when finite field size is considered.
\end{abstract}

\section{INTRODUCTION}

In recent years, multimedia applications such as video conferencing, interactive gaming, voice over IP and cloud computing have become widespread. Unlike traditional applications, these require stringent decoding delays and real-time sequential encoding. Hence, it is of great importance to investigate codes and protocols that take delay into consideration. In this paper, we focus on low delay streaming erasure codes at the application layer. In the ultra-low delay regimes that we consider, block codes are generally not suitable as they have very short block lengths which reduces their error-correction capability.

Low-delay streaming codes for channels that only introduce erasure bursts are studied in [1]. These codes, referred to as Maximally Short (MS) codes, can correct the maximum possible burst-length for a given rate and delay. However MS codes suffer a significant packet loss rate, in the presence of other packet loss patterns. In [2], [3], streaming codes designed for channels that introduce burst and isolated erasures are studied. A tradeoff is established in [2] between the erasure correction capabilities of burst and isolated losses. In [3], a near optimal class of codes - Maximum Distance Span Tradeoff Codes (MIDAS) codes - is proposed which uses two layers of paritychecks, one for optimal burst erasure correction and another for isolated erasures. Another class of codes - Partial Recovery (PRC) codes - that can correct patterns consisting of both burst and isolated erasures in a given window are also proposed. One disadvantage of these codes is that both use StronglyMaximum Distance Separable (SMDS) codes as constituent codes, which has a field size that increases exponentially in the delay [4].
In this paper, we revisit the MIDAS and PRC code constructions with the aim of reducing the field size. We show that by suitably using Maximum Distance Separable (MDS) codes instead of Strongly-MDS codes, the field size is $\mathcal{O}\left(T^{3}\right)$. The MIDAS code with MDS constituent codes are shown to have the same rate as that with Strongly-MDS constituent codes, while PRC code with MDS has a slightly lower rate when compared to its Strongly-MDS version.

\section{Streaming Codes: Definition}

We assume the source packets arrive sequentially at the encoder. In particular, the encoder observes one source symbol $\mathbf{s}[i] \in \mathcal{S}$ at each time $i \geq 0$ and generates a channel packet $\mathbf{x}[i] \in \mathcal{X}$ by causally combining the source symbols,

$$
\mathbf{x}[i]=f_{i}(\mathbf{s}[0], \ldots, \mathbf{s}[i]) .
$$

We consider erasure channels where transmitted symbols are either erased $\mathbf{y}[i]=\star$, or is perfectly received $\mathbf{y}[i]=\mathbf{x}[i]$. The decoder must recover the source symbols within a fixed delay $T$. In particular, for each $i \geq 0$, there exists a decoding function:

$$
\mathbf{s}[i]=g_{i}(\mathbf{y}[0], \ldots, \mathbf{y}[i+T])
$$

The rate of such streaming code is defined by $R=\frac{H(\mathbf{s})}{\log _{2}|\mathcal{X}|}$ is said to be achievable if there exists a feasible code that satisfies (1) and (2). In the subsequent sections, we discuss two families of streaming codes designed for different classes of packet erasures channels and introduce our proposed modified approaches.

\section{MIDAS CODES}

In this section, we consider channels with the following property: in any sliding window of length $W$, the channel can introduce either a burst of length no more than $B$ erasures or a maximum of $N \leq B$ erasures in arbitrary locations. We denote this channel by $\mathcal{C}_{\mathrm{I}}(N, B, W)$. Throughout the analysis of Channel I, we select $W=T+1$ where recall that $T$ is the decoding delay. We first consider an upper bound on the achievable rate, which was presented in [2].

Theorem 1 (Badr et al. [2]). Any achievable rate for $\mathcal{C}_{I}(N, B, T+1)$, satisfies

$$
\left(\frac{R}{1-R}\right) B+N \leq T+1 \text {. }
$$


and furthermore $N \leq B$ and $B \leq T$.

Theorem 1 shows that when the rate $R$ and delay $T$ are fixed there exists a tradeoff between the achievable values of $B$ and $N$. We briefly note two families of codes which are optimal for extreme cases of $B$ and $N$. For the special case of $N=1$, the maximum value of $B$ in (3) is given by $B^{+}=T \cdot \min \left(\frac{1-R}{R}, 1\right)$. It is achievable using Maximally Short Codes (MS) introduced in [1]. For the special case of $B=N$, the maximum value of $N$ in (3) is given by $N^{+}=(1-R)(T+1)$. It can be achieved using Maximum Distance Profile Codes in [5].

For the general choice of parameters $B$ and $N$, Badr et al. [3] proposed a class of codes, MIDAS which generalizes both MS codes and Strongly-MDS codes. MIDAS codes are shown to achieve a near optimal tradeoff between $N$ and $B$. The main steps of this construction is as follows:

- Divide each source symbols $\mathbf{s}[i]$ into two groups $\mathbf{u}[i] \in$ $\mathbb{F}_{q}^{B}$ and $\mathbf{v}[i] \in \mathbb{F}_{q}^{T-B}$.

- Apply a rate $\frac{T-B}{T}$ systematic Strongly-MDS code to the $\mathbf{v}[\cdot]$ symbols generating the parity-check symbols $\mathbf{p}^{v}[\cdot] \in$ $\mathbb{F}_{q}^{B}$.

- Combine the $\mathbf{u}[\cdot]$ symbols with $\mathbf{p}^{v}[\cdot]$ after applying a shift of $T$ to the earlier, i.e., $\mathbf{q}[i]=\mathbf{p}^{v}[i]+\mathbf{u}[i-T]$.

- Apply another systematic Strongly-MDS code of rate $\frac{T-N+1}{T+1}$ to the $\mathbf{u}[\cdot]$ symbols generating the parity-check symbols $\mathbf{p}^{u}[\cdot] \in \mathbb{F}_{q}^{\frac{B N}{T-N+1}}$.

- Concatenate the two streams of parity-check symbols $\mathbf{q}[\cdot]$ and $\mathbf{p}^{u}[\cdot]$ to the source symbols $\mathbf{s}[i]$ to generate the channel symbol,

$$
\mathbf{x}[i]=\left(\mathbf{u}[i], \mathbf{v}[i], \mathbf{q}[i], \mathbf{p}^{u}[i]\right)^{\dagger}
$$

The rate of the code constructed in (4) is $R=\frac{T}{T+B+\frac{N B}{T-N+1}}$. We denote this construction by MIDAS-SMDS. The main disadvantage in the above construction is the use of a StronglyMDS constituent code. While the Strongly-MDS code has a random-linear code type behavior, which is desirable, its required field size [4, Theorem 3.3] grows exponentially in the delay $T$. We propose an alternative construction, MIDASMDS, which replaces Strongly-MDS codes with diagonally interleaved MDS codes. The main advantage of the proposed construction is that the field size grows as $\mathcal{O}\left(T^{3}\right)$. The encoding steps are as follows:

\section{A. Encoding}

- Spit each source symbols $\mathbf{s}[i]$ into $(T-N+1) T$ subsymbols, $\mathbf{s}[i]=\left(s_{0}[i], \ldots, s_{(T-N+1) T-1}[i]\right)$.

- Divide them into two groups,

where

$$
\mathbf{s}[i]=(\mathbf{u}[i], \mathbf{v}[i]),
$$

$$
\begin{aligned}
\mathbf{u}[i] & =\left(u_{0}[i], \ldots, u_{(T-N+1) B-1}[i]\right) \\
& =\left(s_{0}[i], \ldots, s_{(T-N+1) B-1}[i]\right) \\
\mathbf{v}[i] & =\left(v_{0}[i], \ldots, v_{(T-N+1)(T-B)-1}[i]\right) \\
& =\left(s_{(T-N+1) B}[i], \ldots, s_{(T-N+1) T-1}[i]\right) .
\end{aligned}
$$

- Apply a $(T, T-B)$ systematic MDS code to the $\mathbf{v}[\cdot]$ symbols with interleaving factor of $T-N+1$, generating $(T-N+1) B$ parity-check symbols, $\mathbf{p}^{v}[i]=$ $\left(p_{0}^{v}[i], \ldots, p_{(T-N+1) B-1}^{v}[i]\right)$, i.e., the codeword of such MDS code starting at $v_{j}[i]$ is given by,

$$
\mathbf{c}_{j}^{v}[i]=\left[\begin{array}{c}
v_{j}[i] \\
v_{j+(T-N+1)}[i+1] \\
v_{j+2(T-N+1)}[i+2] \\
\vdots \\
v_{j+(T-N+1)(T-B-1)}[i+T-B-1] \\
p_{j}^{v}[i+T-B] \\
p_{j+(T-N+1)}^{v}[i+T-B+1] \\
\vdots \\
p_{j+(T-N+1)(B-1)}^{v}[i+T-1]
\end{array}\right],
$$

for $j=\{0,1, \ldots, T-N\}$.

- Combine the $\mathbf{u}[\cdot]$ symbols with the parity-check symbols $\mathbf{p}^{v}[\cdot]$ after applying a shift of $T$ to the earlier, i.e., $\mathbf{q}[i]=$ $\mathbf{p}^{v}[i]+\mathbf{u}[i-T]$.

- Apply a $(T+1, T-N+1)$ systematic MDS code to the $\mathbf{u}[\cdot]$ symbols with interleaving factor of $B$, generating $B N$ parity-check symbols, $\mathbf{p}^{u}[i]=$ $\left(p_{0}^{u}[i], \ldots, p_{B N-1}^{u}[i]\right)$, i.e., the associated codeword starting at $u_{j}[i]$ is given by,

for $j=\{0,1, \ldots, B-1\}$.

$$
\mathbf{c}_{j}^{u}[i]=\left[\begin{array}{c}
u_{j}[i] \\
u_{j+B}[i+1] \\
u_{j+2 B}[i+2] \\
\vdots \\
u_{j+B(T-N)}[i+T-N] \\
p_{j}^{u}[i+T-N+1] \\
p_{j+B}^{u}[i+T-N+2] \\
\vdots \\
p_{j+B(N-1)}^{u}[i+T]
\end{array}\right]
$$

- Concatenate the parity-check symbols $\mathbf{p}^{u}[\cdot]$ to the previously generated parity-check symbols $\mathbf{q}[\cdot]$, i.e., the channel symbol is given by,

$$
\mathbf{x}[i]=\left(\mathbf{u}[i], \mathbf{v}[i], \mathbf{q}[i], \mathbf{p}^{u}[i]\right)^{\dagger}
$$

\section{B. Decoding}

For the case of burst erasure, we assume the channel introduces an erasure burst of length $B$ in the interval $[i, i+B-1]$. It suffices to show that $\mathbf{s}[i]=(\mathbf{u}[i], \mathbf{v}[i])$ can be recovered by time $T$.

The decoder starts by recovering the parity-check symbols $\mathbf{p}^{\mathrm{I}}[t]$ for $t \in[i+B, i+T-1]$ by subtracting $\mathbf{u}[t-T]$ from $\mathbf{q}[t]$ as they are not erased. However the parity-check symbols $\mathbf{p}^{\mathrm{I}}[t]$ for $t \in[i+T, i+T+B-1]$ are considered not available as they combine $\mathbf{u}[t-T]$ which are erased. One can see that for all $\mathbf{c}_{j}^{v}[r]$ codewords for $r \in\{i-(T-B)+1, \ldots, i+$ $B-1\}$ and $j=\{0,1, \ldots, T-N\}$ spanning the erased $v[\cdot]$ sub-symbols in the interval $[i, i+B-1]$, there are at most $B$ erasures corresponding to symbols falling in the intervals 
$[i, i+B-1]$ and $[i+T, i+T+B-1]$. Therefore, the symbols $\mathbf{v}[i], \ldots, \mathbf{v}[i+B-1]$ can be recovered by time $i+T-1$.

Now, the decoder computes the parity-check symbol $\mathbf{p}^{\mathrm{I}}[i+$ $T]$ as it combines $\mathbf{v}[\cdot]$ which are either not erased or recovered in the previous step and subtract it from $\mathbf{q}[i+T]$ to recover $\mathbf{u}[i]$. This completes the recovery of $\mathbf{s}[i]$ when the channel introduces an erasure burst in the interval $[i, i+B-1]$.

For the isolated erasure capability, we assume the channel introduces $N$ erasures in arbitrary positions in the window $[i, i+T]$. Similar to the burst case, it suffices to show that $\mathbf{s}[i]=(\mathbf{u}[i], \mathbf{v}[i])$ can be recovered by time $T$. The recovery of $\mathbf{u}[i]$ and $\mathbf{v}[i]$ are done separately. For $\mathbf{v}[i]$ it can be verified that all codewords $\mathbf{c}_{j}^{v}[r]$ for $r \in\{i-(T-B)+1, \ldots, i\}$ and $j=\{0,1, \ldots, T-N\}$ have no more than $N \leq B$ erasures. Thus, all sub-symbols of $\mathbf{v}[i]$ are recovered by time $T-1$ since the parity-check symbols $\mathbf{p}^{v}[\cdot]$ in the interval $[0, T-1]$ are available. For $\mathbf{u}[i]$, we consider the codewords $\mathbf{c}_{j}^{u}[r]$ for $r \in\{i-(-N), \ldots, i\}$ and $j=\{0,1, \ldots, B-1\}$. Similarly, all considered codewords have no more than $N$ erasures and thus $\mathbf{u}[i]$ can be recovered by time $T$ and the claim follows.

We note that for a given pair $(N, B)$ and a delay $T$, both MIDAS-SMDS and MIDAS-MDS codes achieve the same rate $R=\frac{T}{T+B+\frac{N B}{T-N+1}}$. However as we argue next, MIDAS-MDS code requires a much smaller field-size in general. Splitting each source symbol into $(T-N+1) T$ sub-symbols requires that each source symbol consist of $q_{1}=(T-N+1) T$ subsymbols. We therefore need to determine the field size of each sub-symbol. Using the well-known fact that an $(n, k) \mathrm{MDS}$ codes exists for any field-size which is a prime number greater than $n$ we note that the field size needed for both $(T, T-B)$ and $(T+1, T-N+1)$ MDS codes to exist is $q_{2}=p(T+1)$ where $p(n)$ is the smallest prime greater than $n$ are known to exist. Thus the required field size is $q=q_{1} \cdot q_{2}$.

\section{Example}

Table I illustrates a MIDAS-MDS construction for $(N, B)=(2,3)$ and $T=4$ achieving a rate of $R=$ $\frac{T}{T+B+\frac{N B}{T-N+1}}=\frac{4}{9}$. The encoding steps are as follows,

- Split each source symbol $\mathbf{s}[i]$ into $(T-N+1) T=12$ sub-symbols. The first $(T-N+1) B=9$ of which are $\left(u_{0}[i], \ldots, u_{8}[i]\right)$ while the last $(T-N+1)(T-B)=3$ are $\left(v_{0}[i], v_{1}[i], v_{2}[i]\right)$.

- Apply a $(T, T-B)=(4,1)$ MDS code to the $v$ subsymbols with an interleaving factor of $T-N+1=3$ as shown using the shaded boxed in Table I. This generates $(T-N+1) B=9$ parity-check sub-symbols $\left(p_{0}^{v}[i], \ldots, p_{8}^{v}[i]\right)$.

- Combine the $u$ symbols $u_{0}, \ldots, u_{8}$ with the generated parity-check symbols $p^{v}$ after applying a shift of $T$ units to the former.

- Apply a $(T+1, T-N+1)=(5,3)$ MDS code to the $u$ symbols with an interleaving factor of $B=3$ generating $B N=6$ parity-check sub-symbols $\left(p_{0}^{u}[i], \ldots, p_{5}^{u}[i]\right)$ as illustrated by the white boxes in Table. I.

The decoding steps are straight forward by following all codewords spanning the erased symbols.

\begin{tabular}{|c|c|c|c|c|}
\hline$[i]$ & {$[i+1]$} & {$[i+2]$} & {$[i+3]$} & {$[i+4]$} \\
\hline$u_{0}[i]$ & $u_{0}[i+1]$ & $u_{0}[i+2]$ & $u_{0}[i+3]$ & $u_{0}[i+4]$ \\
\hline $\begin{array}{l}u_{1}[i] \\
u_{2}[i]\end{array}$ & $\begin{array}{l}u_{1}[i+1] \\
u_{2}[i+1]\end{array}$ & $\begin{array}{l}u_{1}[i+2] \\
u_{2}[i+2]\end{array}$ & $\begin{array}{l}u_{1}[i+3] \\
u_{2}[i+3]\end{array}$ & $\begin{array}{l}u_{1}[i+4] \\
u_{2}[i+4]\end{array}$ \\
\hline$u_{3}[i]$ & $u_{3}[i+1]$ & $u_{3}[i+2]$ & $u_{3}[i+3]$ & $u_{3}[i+4]$ \\
\hline $\begin{array}{l}u_{4}[i] \\
u_{5}[i]\end{array}$ & $\begin{array}{l}u_{4}[i+1] \\
u_{5}[i+1]\end{array}$ & $\begin{array}{l}u_{4}[i+2] \\
u_{5}[i+2]\end{array}$ & $\begin{array}{l}u_{4}[i+3] \\
u_{5}[i+3]\end{array}$ & $\begin{array}{l}u_{4}[i+4] \\
u_{5}[i+4]\end{array}$ \\
\hline$u_{6}[i]$ & $u_{6}[i+1]$ & $u_{6}[i+2]$ & $u_{6}[i+3]$ & $u_{6}[i+4]$ \\
\hline $\begin{array}{l}u_{7}[i] \\
u_{8}[i]\end{array}$ & $\begin{array}{l}u_{7}[i+1] \\
u_{8}[i+1]\end{array}$ & $\begin{array}{l}u_{7}[i+2] \\
u_{8}[i+2]\end{array}$ & $\begin{array}{l}u_{7}[i+3] \\
u_{8}[i+3]\end{array}$ & $\begin{array}{l}u_{7}[i+4] \\
u_{8}[i+4]\end{array}$ \\
\hline$v_{0}[i]$ & $v_{0}[i+1]$ & $v_{0}[i+2]$ & $v_{0}[i+3]$ & $v_{0}[i+4]$ \\
\hline $\begin{array}{l}v_{1}[i] \\
v_{2}[i] \\
\end{array}$ & $\begin{array}{l}v_{1}[i+1] \\
v_{2}[i+1]\end{array}$ & $\begin{array}{l}v_{1}[i+2] \\
v_{2}[i+2]\end{array}$ & $\begin{array}{l}v_{1}[i+3] \\
v_{2}[i+3] \\
\end{array}$ & $\begin{array}{l}v_{1}[i+4] \\
v_{2}[i+4] \\
\end{array}$ \\
\hline$p_{0}^{v}[i]$ & $p_{0}^{v}[i+1]$ & $p_{0}^{v}[i+2]$ & $p_{0}^{v}[i+3]$ & $p_{0}^{v}[i+4]$ \\
\hline $\begin{array}{l}p_{1}^{v}[i] \\
p_{2}^{v}[i]\end{array}$ & $\begin{array}{l}p_{1}^{v}[i+1] \\
p_{2}^{v}[i+1]\end{array}$ & $\begin{array}{l}p_{1}^{v}[i+2] \\
p_{2}^{v}[i+2]\end{array}$ & $\begin{array}{l}p_{1}^{v}[i+3] \\
p_{2}^{v}[i+3]\end{array}$ & $\begin{array}{l}p_{1}^{v}[i+4] \\
p_{2}^{v}[i+4]\end{array}$ \\
\hline$p_{3}^{v}[i]$ & $p_{3}^{v}[i+1]$ & $p_{3}^{v}[i+2]$ & $p_{3}^{v}[i+3]$ & $p_{3}^{v}[i+4]$ \\
\hline $\begin{array}{l}p_{4}^{v}[i] \\
p_{5}^{v}[i]\end{array}$ & $\begin{array}{l}p_{4}^{v}[i+1] \\
p_{5}^{v}[i+1]\end{array}$ & $\begin{array}{l}p_{4}^{v}[i+2] \\
p_{5}^{v}[i+2]\end{array}$ & $\begin{array}{l}p_{4}^{v}[i+3] \\
p_{5}^{v}[i+3]\end{array}$ & $\begin{array}{l}p_{4}^{v}[i+4] \\
p_{5}^{v}[i+4]\end{array}$ \\
\hline$p_{6}^{v}[i]$ & $p_{6}^{v}[i+1]$ & $p_{6}^{v}[i+2]$ & $p_{6}^{v}[i+3]$ & $p_{6}^{v}[i+4]$ \\
\hline $\begin{array}{l}p_{7}^{v}[i] \\
p_{8}^{v}[i]\end{array}$ & $\begin{array}{l}p_{7}^{v}[i+1] \\
p_{8}^{v}[i+1]\end{array}$ & $\begin{array}{l}p_{7}^{v}[i+2] \\
p_{8}^{v}[i+2]\end{array}$ & $\begin{array}{l}p_{7}^{v}[i+3] \\
p_{8}^{v}[i+3]\end{array}$ & $\begin{array}{l}p_{7}^{v}[i+4] \\
p_{8}^{v}[i+4]\end{array}$ \\
\hline$p_{0}^{u}[i]$ & $p_{0}^{u}[i+1]$ & $p_{0}^{u}[i+2]$ & $p_{0}^{u}[i+3]$ & $p_{0}^{u}[i+4]$ \\
\hline $\begin{array}{l}p_{1}^{u}[i] \\
p_{2}^{u}[i]\end{array}$ & $\begin{array}{l}p_{1}^{u}[i+1] \\
p_{2}^{u}[i+1]\end{array}$ & $\begin{array}{l}p_{1}^{u}[i+2] \\
p_{2}^{u}[i+2]\end{array}$ & $\begin{array}{l}p_{1}^{u}[i+3] \\
p_{2}^{u}[i+3]\end{array}$ & $\begin{array}{l}p_{1}^{u}[i+4] \\
p_{2}^{u}[i+4]\end{array}$ \\
\hline$p_{3}^{u}[i]$ & $p_{3}^{u}[i+1]$ & $p_{3}^{u}[i+2]$ & $p_{3}^{u}[i+3]$ & $p_{3}^{u}[i+4]$ \\
\hline $\begin{array}{l}p_{4}^{u}[i] \\
p_{5}^{u}[i]\end{array}$ & $\begin{array}{l}p_{4}^{u}[i+1] \\
p_{5}^{u}[i+1]\end{array}$ & $\begin{array}{l}p_{4}^{u}[i+2] \\
p_{5}^{u}[i+2]\end{array}$ & $\begin{array}{l}p_{4}^{u}[i+3] \\
p_{5}^{u}[i+3]\end{array}$ & $\begin{array}{l}p_{4}^{u}[i+4] \\
p_{5}^{u}[i+4]\end{array}$ \\
\hline
\end{tabular}

TABLE I: MIDAS-MDS code construction for $(N, B)=$ $(2,3)$, a delay of $T=4$ and rate $R=4 / 9$. We note that each of the parity-check sub-symbols $p_{j}^{v}[t]$ is combined with $u_{j}[t-4]$ for $j=\{0,1, \ldots, 8\}$ but are omitted for simplicity.

\section{PRC CODES}

Partial Recovery Codes (PRC) considered in this section are designed for the channel with the following property: in any sliding window of length $W$, the channel can introduce no more than a burst of maximum length $B$ and one isolated erasure (before or after the burst). We denote such channel by $\mathcal{C}_{\text {II }}(B, W)^{1}$. The term "partial recovery" is used because these codes do not attempt to recover every erased source packet, but only recover a subset. In [3], PRC codes are studied for the special case when $W=\infty$. The channel can introduce one erasure burst and one isolated erasure anywhere either before or after the burst. We summarize the proposed construction below:

- Divide each source symbols $\mathbf{s}[i]$ into two groups $\mathbf{u}[i] \in$ $\mathbb{F}_{q}^{u}$ and $\mathbf{v}[i] \in \mathbb{F}_{q}^{v}$ where $u=(B+1)(T-\Delta+1)-(\Delta-$ $B-1)$ and $v=(T-\Delta+1)(\Delta-B-1)$.

- Apply a rate $\frac{v}{v+u}$ systematic Strongly-MDS code to the $\mathbf{v}[\cdot]$ symbols generating the parity-check symbols $\mathbf{p}^{\mathrm{I}}[\cdot] \in$ $\mathbb{F}_{q}^{u}$.

- Combine the $\mathbf{u}[\cdot]$ symbols with the parity-check symbols $\mathbf{p}^{\mathrm{I}}[\cdot]$ after applying a shift of $\Delta \leq T$ to the $\mathbf{u}[\cdot]$ symbols,

\footnotetext{
${ }^{1}$ Codes designed for channel $\mathcal{C}_{\mathrm{II}}(B, W)$ can be modified to correct isolated erasures by adding an extra layer of parity-check symbols similar to that in the MIDAS construction
} 
i.e., $\mathbf{q}[i]=\mathbf{p}^{\mathrm{I}}[i]+\mathbf{u}[i-\Delta]$.

- Apply another systematic Strongly-MDS code of rate $\frac{v}{v+s}$ to the $\mathbf{v}[\cdot]$ symbols which generates the parity-check symbols $\mathbf{p}^{\mathrm{II}}[\cdot] \in \mathbb{F}_{q}^{s}$ where $s=\Delta-B-1$.

- Concatenate the two streams of parity-check symbols $\mathbf{p}^{\mathrm{I}}[\cdot]$ and $\mathbf{p}^{\mathrm{II}}[\cdot]$ to the source symbols $\mathbf{s}[i]$ to generate the channel symbol,

$$
\mathbf{x}[i]=\left(\mathbf{u}[i], \mathbf{v}[i], \mathbf{q}[i], \mathbf{p}^{\mathrm{II}}[i]\right)^{\dagger}
$$

We refer to the construction in (11) as PRC-SMDS code. Such construction achieves a rate of,

$$
\begin{aligned}
R & =\frac{u+v}{2 u+v+s} \\
& =\frac{\Delta(T-\Delta)+(B+1)}{\Delta(T-\Delta)+(B+1)(T-\Delta+2)}
\end{aligned}
$$

Maximizing $R$ by finding the optimal $\Delta$, one gets,

$$
R^{\star}=\frac{(T+2) \sqrt{T-B}-2(T-B)}{(T+B+3) \sqrt{T-B}-2(T-B)} .
$$

As with the case of MIDAS codes, we propose a construction which replaces Strongly-MDS codes in the PRC-SMDS construction with MDS codes. We denote this construction by PRC-MDS code. The detailed encoding steps are as follows:

\section{A. Encoding}

- Spit each source symbols $\mathbf{s}[i]$ into $(T-\Delta+1) \Delta$ subsymbols, $\mathbf{s}[i]=\left(s_{0}[i], \ldots, s_{(T-\Delta+1) \Delta-1}[i]\right)$.

- Divide them into two groups,

$$
\begin{aligned}
& \text { where } \\
& \begin{aligned}
\mathbf{u}[i] & =\left(u_{0}[i], \ldots, u_{(T-\Delta+1)(B+1)-1}[i]\right) \\
& =\left(s_{0}[i], \ldots, s_{(T-\Delta+1)(B+1)-1}[i]\right) \\
\mathbf{v}[i] & =\left(v_{0}[i], \ldots, v_{(T-\Delta+1)(\Delta-B-1)-1}[i]\right) \\
& =\left(s_{(T-\Delta+1)(B+1)}[i], \ldots, s_{(T-\Delta+1) \Delta-1}[i]\right) .
\end{aligned}
\end{aligned}
$$

$$
\mathbf{s}[i]=(\mathbf{u}[i], \mathbf{v}[i])
$$

- Apply a systematic $(\Delta, \Delta-B-1)$ MDS code to the $\mathbf{v}[\cdot]$ symbols with interleaving factor of $T-\Delta+1$, generating $(T-\Delta+1)(B+1)$ parity-check symbols, $\mathbf{p}^{\mathrm{I}}[\cdot]=\left(p_{0}^{\mathrm{I}}[i], \ldots, p_{(T-\Delta+1)(B+1)-1}^{\mathrm{I}}[i]\right)$. The resulting codeword of the such MDS code starting at $v_{j}[i]$ is given by,

$$
\mathbf{c}_{j}^{\mathrm{I}}[i]=\left[\begin{array}{c}
v_{j}[i] \\
v_{j+(T-\Delta+1)}[i+1] \\
v_{j+2(T-\Delta+1)}[i+2] \\
\vdots \\
v_{j+(T-\Delta+1)(\Delta-B-2)}[i+\Delta-B-2] \\
p_{j}^{\mathrm{I}}[i+\Delta-B-1] \\
p_{j+(T-\Delta+1)}^{\mathrm{I}}[i+\Delta-B] \\
\vdots \\
p_{j+(T-\Delta+1) B}^{\mathrm{I}}[i+\Delta-1]
\end{array}\right],
$$

for $j=\{0,1, \ldots, T-\Delta\}$.

- Combine the $\mathbf{u}[\cdot]$ symbols with the parity-check symbols $\mathbf{p}^{\mathrm{I}}[\cdot]$ after applying a shift of $\Delta$ to the earlier, i.e., $\mathbf{q}[i]=$ $\mathbf{p}^{\mathrm{I}}[i]+\mathbf{u}[i-\Delta]$

- Apply a systematic $(T-\Delta+2, T-\Delta+1)$ MDS code to the $\mathbf{v}[\cdot]$ symbols with interleaving factor of $\Delta-B-1$, generating $\Delta-B-1$ parity-check symbols, $\mathbf{p}^{\mathrm{II}}[\cdot]=$ $\left(p_{0}^{\mathrm{II}}[i], \ldots, p_{(\Delta-B-1)-1}^{\mathrm{II}}[i]\right)$, i.e., the resulting codeword would be,

$$
\mathbf{c}_{j}^{\mathrm{II}}[i]=\left[\begin{array}{c}
v_{j}[i] \\
v_{j+(\Delta-B-1)}[i+1] \\
v_{j+2(\Delta-B-1)}[i+2] \\
\vdots \\
v_{j+(\Delta-B-1)(T-\Delta)}[i+T-\Delta] \\
p_{j}^{\mathrm{I}}[i+T-\Delta+1]
\end{array}\right],
$$

for $j=\{0,1, \ldots, \Delta-B-2\}$.

- Concatenate the parity-check symbols $\mathbf{p}^{\mathrm{II}}[\cdot]$ to the previously generated parity-check symbols $\mathbf{q}[\cdot]$, i.e., the channel symbol is given by,

$$
\mathbf{x}[i]=\left(\mathbf{s}[i], \mathbf{q}[i], \mathbf{p}^{\mathrm{II}}[i]\right) .
$$

One can see that the rate of the constructed code in (19) is given by,

$$
R=\frac{(T-\Delta+1) \Delta}{(T-\Delta+1)(\Delta+B+1)+(\Delta-B-1)} .
$$

Similar to the case of PRC-SMDS codes, the optimal value of $\Delta$ (ignoring integer effects) for maximizing the rate $R$ can be shown to be:

$$
\Delta^{\star}=\frac{T(B+1)-\sqrt{T(B+1)(T-B)}}{B} .
$$

\section{B. Decoding}

We assume the channel introduces an erasure burst of length $B$ in the interval $[i, i+B-1]$ together with an isolated erasure happening at time $t_{i}$. We consider the following cases:

- $t_{i} \in[i-T, i-(T-\Delta)-1]$ : In this case, the $\mathbf{v}\left[t_{i}\right]$ can be recovered using the parity-check symbols $\mathbf{p}^{\mathrm{II}}[\cdot]$ of the $(T-\Delta+2, T-\Delta+1)$ MDS code by time $t_{i}+(T-$ $\Delta+2)-1 \leq i$. This is because the codewords $\mathbf{c}_{j}^{\mathrm{II}}[r]$ for $r=\left\{t_{i}-T+\Delta, \ldots, t_{i}\right\}$ and $j=\{0,1, \ldots, \Delta-B-$ $2\}$ have no more than one erasure. Now, the symbols $\mathbf{v}[i], \ldots, \mathbf{v}[i+B-1]$ can be recovered using the paritycheck symbols $\mathbf{p}^{\mathrm{I}}[\cdot]$ of the $(\Delta, \Delta-B-1)$ MDS code by time $i+\Delta-1$. Thus the symbols $\mathbf{u}[i], \ldots, \mathbf{u}[i+$ $B-1]$ can be recovered at time $i+\Delta, \ldots, i+\Delta+$ $B-1$ sequentially by subtracting the corresponding $\mathbf{p}^{\mathrm{II}}[\cdot]$ parity-check symbols. We note that if $t_{i}+\Delta \notin[i, i+$ $B-1]$, one can recover $\mathbf{u}\left[t_{i}\right]$, otherwise it is lost.

- $t_{i} \in[i-(T-\Delta), i-1]$ : Here, both the $\mathbf{v}[\cdot]$ symbols of isolated erasure together with that of the erasure burst are recovered using the $(\Delta, \Delta-B-1)$ MDS code. In particular, $\mathbf{v}\left[t_{i}\right]$ is recovered by time $t_{i}+\Delta-1$ using the associated MDS code, while $\mathbf{v}[i], \ldots, \mathbf{v}[i+B-1]$ are all guaranteed to be recovered by time $i+\Delta-1$ as explained in the MIDAS decoder. Now, the $\mathbf{u}[\cdot]$ symbols can be recovered sequentially at time $i+\Delta, \ldots, i+\Delta+B-1$.

- $t_{i} \in[i+B, i+\Delta-1]$ : The $\mathbf{v}[\cdot]$ symbols of the erasure burst can be recovered using the $(\Delta, \Delta-B-1)$ MDS code at time $i+\Delta-1$ since there is no more than $B+1$ erasures in each of the codewords, $\mathbf{c}_{j}^{\mathrm{I}}[r]$ for $r=\{i-$ $\Delta+B+2, \ldots, i+B-1\}$ and $j=\{0,1, \ldots, T-\Delta\}$. The $\mathbf{v}\left[t_{i}\right]$ symbol can now be recovered using the $(T-$ 


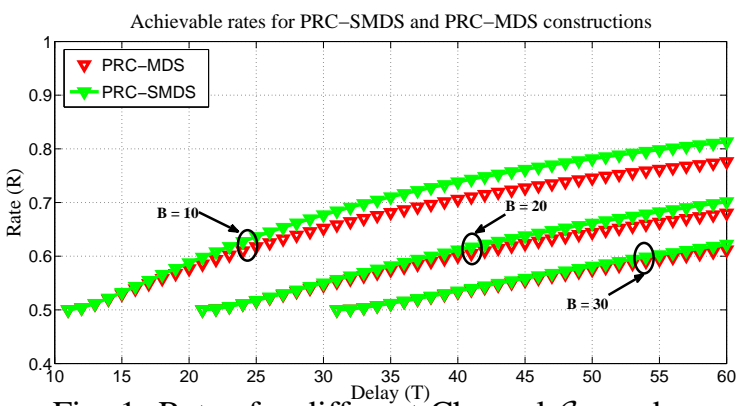

Fig. 1: Rates for different Channel $\mathcal{C}_{\text {II }}$ codes.

$\Delta+2, T-\Delta+1)$ code by time $t_{i}+T-\Delta+1 \leq$ $i+\Delta-1+T-\Delta+1=i+T$ since all $\mathbf{v}[\cdot]$ symbols are recovered except for one. Now, the decoder can go back and compute the parity-check symbols $\mathbf{p}^{\mathrm{I}}[\cdot]$ in the interval $[i+\Delta, i+\Delta+B-1]$ and subtract them to compute $\mathbf{u}[i], \ldots, \mathbf{u}[i+B-1]$. Also, $\mathbf{u}\left[t_{i}\right]$ can be recovered later at time $t_{i}+\Delta$.

- $t_{i} \in[i+\Delta, i+\Delta+B-1]$ : Similar to the previous case, the $\mathbf{v}[\cdot]$ symbols of the erasure burst are recovered by time $i+\Delta-1$ since the codewords. Now the paritycheck symbols $\mathbf{p}^{\mathrm{I}}[\cdot]$ in the interval $\left[i+\Delta, t_{i}-1\right]$ can be computed and subtracted to recover $\mathbf{u}[i], \ldots, \mathbf{u}\left[t_{i}-\right.$ $\Delta-1]$. The $\mathbf{v}\left[t_{i}\right]$ symbol can be recovered using the $(T-\Delta+2, T-\Delta+1)$ by time $t_{i}+(T-\Delta)+1$. At this time the decoder goes back to compute the parity-check symbols $\mathbf{p}^{\mathrm{I}}[\cdot]$ in the interval $\left[t_{i}+1, i+\Delta+B-1\right]$ and subtract them to recover $\mathbf{u}\left[t_{i}-\Delta+1\right], \ldots, \mathbf{u}[i+B-1]$. Also, $\mathbf{u}\left[t_{i}\right]$ can be recovered later at time $t_{i}+\Delta$. We note that $\mathbf{u}\left[t_{i}-\Delta\right]$ can not be recovered since the isolated erasure at time $t_{i}$ erases its repeated version.

Unlike the case of MIDAS codes, PRC-MDS codes has slightly lower rate than PRC-SMDS codes for a given $B$ and $T$. Fig. 1 shows the achieved rates of both codes for $B=10$, 20 and 30 and different values of $T$. The gap between the achieved rates of both codes increases with $T$ but decreases with increasing $B$.

Computing the sufficient field size for constructing PRCMDS codes is similar to that of MIDAS-MDS codes. The source vector must consist of $q_{1}=(T-\Delta+1) \Delta$ sub-symbols. Also, the $(\Delta, \Delta-B-1)$ and $(T-\Delta+2, T-\Delta+1)$ MDS codes can be constructed if the field size $q_{2}=p(\max (\Delta, T-$ $\Delta+2)$ ). Thus, for a given pair $B$ and a delay $T$, a field size of $q=q_{1} \cdot q_{2}$ - grows as $\mathcal{O}\left(T^{3}\right)$ - is sufficient to construct the corresponding PRC-MDS code.

\section{Performance in Non-Ideal Erasure Patterns}

In this section, we give some qualitative insights on why MIDAS and PRC with MDS constituent codes in fact have an inferior performance than their Strongly-MDS versions in simulation over Gilbert-Elliott channels. We explain this by considering specific erasure patterns.

\section{A. MIDAS Codes}

We argue that when the channel introduces a burst and one isolated erasure, MIDAS-MDS codes are more sensitive when compared to MIDAS-SMDS codes. We illustrate this

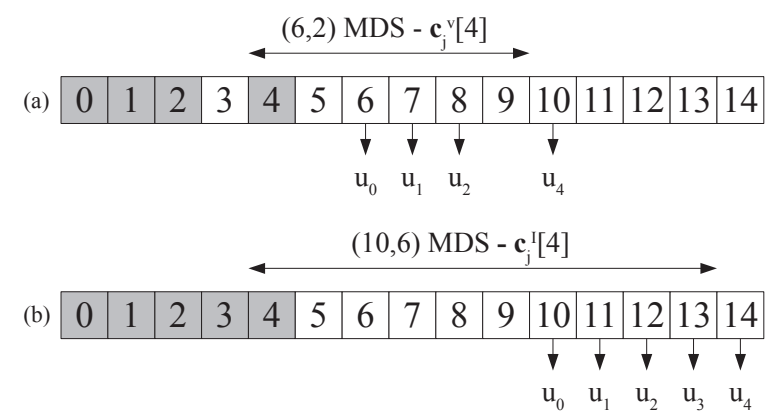

Fig. 2: Non-ideal erasure patterns for (a) MIDAS and (b) PRC codes.

argument through the following example. We consider both versions of MIDAS codes with $(N, B)=(2,4)$ and $T=6$. If the actual channel introduces a burst of length 3 in the interval $[0,2]$ and one isolated erasure at time 4 as shown in Fig. 2, a MIDAS-SMDS code can recover all erasues $\mathbf{v}[\cdot]$ symbols by time 5 and then recover the $\mathbf{u}[\cdot]$ symbols sequentially and perfect recovery follows. While in the MIDAS-MDS version, the $(T, T-B)=(6,2)$ MDS recovers $\mathbf{v}[4]$ at time 9 as shown in Fig. 2. This is already after the deadline of $\mathbf{s}[0], \mathbf{s}[1]$ and $\mathbf{s}[2]$ thus these are not recovered. More generally, when the channel introduces a burst of length $B_{p}$ in the interval $\left[0, B_{p}-1\right]$ where $N<B_{p}<B$ and an isolated erasure in the interval $\left[B_{p}+1, T-\lceil T / B\rceil\right]$ MIDAS-SMDS will recover all the erased symbols whereas MIDAS-MDS will fail to recover any of the erased symbols in the burst.

\section{B. PRC Codes}

We argue that PRC-SMDS codes are more robust when compared to PRC-MDS codes when the channel introduces bursts longer than the targeted value of $B$. Consider both PRCSMDS and PRC-MDS codes with $B=3$ and $T=12$ and a channel introducing a burst of length $5>B+1=4$ in the interval $[0,4]$ as illustrated in Fig. 2. It can be shown that the value of $\Delta$ for both codes is 10 . For the Strongly-MDS version, the decoder will use parity-check symbols at time 15 together with those in the interval $[5,9]$ and the $\mathbf{p}^{\mathrm{II}}[\cdot]$ in the interval $[10,14]$ to recover the erased $\mathbf{v}[\cdot]$ symbols. Now (at time 15), the decoder can recover $\mathbf{u}[0], \ldots, \mathbf{u}[4]$ within delays of $\{15,14,13,12,11\}$, respectively, i.e., a partial recovery of 2 symbols, $\mathbf{s}[3]$ and $\mathbf{s}[4]$. On the other hand, PRC-MDS fails to recover $\mathbf{v}[4]$ as the associated diagonal $\mathbf{c}_{j}^{\mathrm{I}}[4]$ has more than $B+1$ erasures at time $t \in\{4,10,11,12,13\}$. Hence, the entire erasure burst is lost.

\section{Simulation Results}

We consider a Gilbert-Elliott channel model with two states, bad-state and good-state. The probability that the symbol is lost in the bad-state is 1 while that in the good-state is $\varepsilon$. In other words, the channel introduces bursts in the bad-state, i.i.d. erasures in the good-state and both in the transition from bad-state to good-state or vice versa.

The Channel and Code parameters used in both experiments are given in Table. II. 

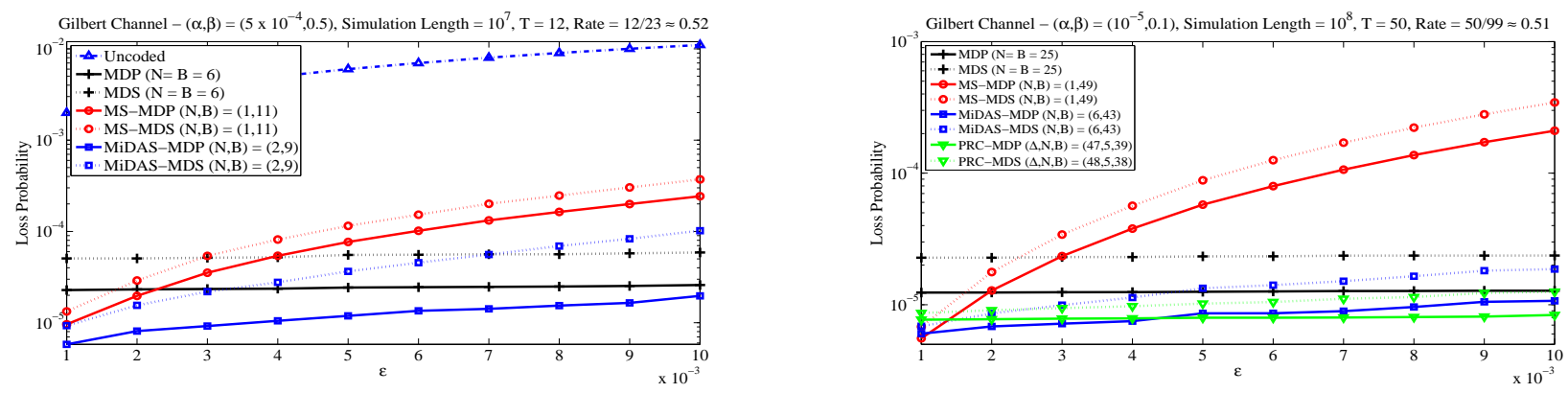

(a) Simulation over a Gilbert-Elliott Channel with $(\alpha, \beta)=\left(5 \times 10^{-4}, 0.5\right)$. (b) Simulation over a Gilbert-Elliott Channel with $(\alpha, \beta)=\left(10^{-5}, 0.1\right)$. All All codes are evaluated using a decoding delay of $T=12$ symbols and a rate codes are evaluated using a decoding delay of $T=50$ symbols and a rate of of $R=12 / 23 \approx 0.52$. $R=50 / 99 \approx 0.51$

Fig. 3: Simulation Experiments for Gilbert-Elliott Channel Model with different parameters.

\begin{tabular}{|c|c|c|c|c|}
\hline \multirow{6}{*}{$\begin{array}{l} \\
(\alpha, \beta) \\
\text { Channel Length } \\
\text { Rate } R \\
\text { Delay } T \\
\end{array}$} & \multicolumn{2}{|c|}{ Fig. 3(a) } & \multicolumn{2}{|c|}{ Fig. 3(b) } \\
\hline & \multirow{4}{*}{\multicolumn{2}{|c|}{$\begin{array}{c}\left(5 \times 10^{-4}, 0.5\right) \\
10^{7} \\
12 / 23 \approx 0.52 \\
12\end{array}$}} & \multirow{4}{*}{\multicolumn{2}{|c|}{$\begin{array}{c}\left(5 \times 10^{-5}, 0.1\right) \\
10^{8} \\
50 / 99 \approx 0.51 \\
50\end{array}$}} \\
\hline & & & & \\
\hline & & & & \\
\hline & & & & \\
\hline & $\mathrm{N}$ & $\mathrm{B}$ & $\mathrm{N}$ & $\mathrm{B}$ \\
\hline Strongly-MDS & 6 & 6 & 25 & 25 \\
\hline MDS & 6 & 6 & 25 & 25 \\
\hline MS-SMDS & 1 & 11 & 1 & 49 \\
\hline MS-MDS & 1 & 11 & 1 & 49 \\
\hline MIDAS-SMDS & 2 & 9 & 6 & 43 \\
\hline MIDAS-MDS & 2 & 9 & 6 & 43 \\
\hline PRC-SMDS & - & - & 5 & 39 \\
\hline PRC-MDS & - & - & 5 & 38 \\
\hline
\end{tabular}

TABLE II: Channel and Code Parameters used in Simulations.

In Fig. 3(a) and Fig. 3(b), we plot the loss rate on the yaxis versus $\varepsilon$ on the $\mathrm{x}$-axis. The loss rate of the Strongly-MDS version of all codes is shown in solid lines while that of the MDS version of the same codes is shown in dashed lines. The performance of various codes is as follows:

- Strongly-MDS (Maximum $N$ ) Codes - Black Lines As discussed earlier, Strongly-MDS codes achieve the maximum value of $N$ for a given rate $R$ and delay $T$. Another way of achieving the same $N$ is splitting each source symbol into $T-N+1$ sub-symbols and applying a $(T+1, T-N+1)$ MDS code along the diagonals. Due to the large value of $N$ for both codes, they can recover from all erasures in the good-state which explains the independence on $\varepsilon$ in the interval of interest. The loss probability of these codes is determined by the bursts of length longer than $N$. However, one can see that Strongly-MDS codes has a slightly better performance which is due to its capability of partial recovery when the channel introduces bursts longer than $N$.

- Maximally Short (Maximum B) Codes - Red Lines Maximally Short (MS) codes can be considered as a special case of MIDAS codes with no $\mathbf{p}^{u}[\cdot]$ parity-check symbols added. One can see that for both MS-SMDS and MS-MDS codes, there is a noticeable increase in the loss rate in the interval of $\varepsilon$ considered. The packet loss probability increases in proportion to $\varepsilon^{2}$ as $N=1$ for these codes.

\section{- MIDAS Codes - Blue Lines}

With choosing the right pair $(N, B)$, the MIDAS codes has a larger $B$ compared to maximum $N$ codes and larger $N$ compared to maximum $B$ codes. Thus, they achieve a better performance than both codes for some values of $\varepsilon$. However, the performance deteriorates faster than that of Strongly-MDS codes. This is because of the bad performance of MIDAS codes against burst plus isolated erasure patterns introduces by the Gilbert-Elliott channel in the transition from bad to good states and vice versa as discussed in section V. However, the performance of MIDAS-MDS deteriorates slightly faster than MIDASSMDS codes which is due to an even worse performance when facing a subset of the burst plus isolated erasure patterns (c.f. V).

\section{- PRC Codes - Green Lines}

PRC codes are designed to recover from burst and isolated erasures with a maximum loss of one symbol. This explains the slower deterioration in performance when compared to MIDAS codes. We note that PRC codes are not plotted in Fig. 3(a) as for $R=12 / 23$ and $T=12$ and $N \geq 2$, a PRC code achieves $B \leq 6$ which is what an MDS or Strongly-MDS code achieves in this case. Thus, the performance of PRC codes is expected to be close to these constructions. The PRC-SMDS codes also outperform PRC-MDS codes due to the better partial recovery property discussed before as well as the slightly larger value of $B$ in Table II.

\section{REFERENCES}

[1] E. Martinian and C.-E. W. Sundberg, "Burst erasure correction codes with low decoding delay," IEEE Transactions on Information Theory, vol. 50, no. 10, pp. 2494-2502, 2004.

[2] A. Badr, A. Khisti, W. Tan, and J. Apostolopoulos, "Streaming codes for channels with burst and isolated erasures," in INFOCOM, 2013.

[3] — - "Robust streaming erasure codes based on deterministic channel approximations," in ISIT, Istanbul, Turkey, July 2013.

[4] H. Gluesing-Luerssen, J. Rosenthal, and R. Smarandache, "On superregular matrices and mdp convolutional codes," Linear Algebra and its Applications, vol. 428, no. 11, pp. 2585-2596, 2008.

[5] V. Tomas, J. Rosenthal, and R. Smarandache, "Decoding of convolutional codes over the erasure channel," IEEE Transactions on Information Theory, vol. 58, no. 1, pp. 90-108, 2012. 